\title{
PROMOÇÃO DA QUALIDADE DE VIDA NO TRABALHO: RELATO DE EXPERIÊNCIA DE UM WORKSHOP ENVOLVENDO OS TRABALHADORES DE ENFERMAGEM
}

\author{
Anna Cláudia Maurício Telles , Patrícia Campos Pavan Baptista², Lucinda Helena da Silva de Vasconcelos ${ }^{3}$, Carolina Luiza
} Bernardes ${ }^{4}$, Ana Paula Pelegrini Ratier ${ }^{5}$

${ }^{1}$ Enfermeira. Mestre Enfermagem. Hospital Universitário de São Paulo. São Paulo, SP, Brasil.

${ }^{2}$ Enfermeira. Pós-doutorado em Enfermagem. Docente da Escola de Enfermagem da Universidade de São Paulo. São Paulo, SP, Brasil.

${ }^{3}$ Fisioterapeuta. Mestre em Enfermagem. Hospital Universitário da Universidade de São Paulo. São Paulo, SP, Brasil.

${ }^{4}$ Enfermeira. Mestre em Enfermagem. Docente da Faculdade Oswaldo Cruz. São Paulo, SP, Brasil.

${ }^{5}$ Terapeuta Ocupacional. Doutoranda em Ciências Sociais. Escola de Enfermagem da Universidade de São Paulo. São Paulo, SP, Brasil.

RESUMO: O objetivo do relato é descrever a experiência da realização de um workshop direcionado à Promoção da Qualidade de Vida no Trabalho com trabalhadores de enfermagem. Trata-se de relato de experiência sobre a realização de um workshop com trabalhadores de enfermagem de um hospital universitário. Participaram do workshop 60 trabalhadores de enfermagem em seis estações de trabalho destinadas à Promoção da Qualidade de Vida no Trabalho. Foram realizadas seis estações de trabalho, simultaneamente, com aproximadamente 10 trabalhadores em cada uma e duração média de 30 minutos. As estações foram conduzidas por enfermeiros e fisioterapeutas com o apoio de docente e temáticas relevantes para a Saúde do trabalhador. O relato visa contribuir para ampliação das discussões sobre o tema, oferecendo subsídios para repensar o processo de trabalho e as possíveis intervenções com vistas à melhoria das condições de trabalho, empoderamento dos trabalhadores no tocante ao autocuidado e prevenção de acidentes e doenças.

DESCRITORES: Enfermagem; Saúde do trabalhador; Promoção da saúde.

\section{PROMOTING QUALITY OF LIFE AT WORK: EXPERIENCE REPORT OF A WORKSHOP INVOLVING NURSING STAFF}

\begin{abstract}
The aim of the report is to describe the experience of a workshop focused on the Promotion of Quality of Life at Work with nursing staff. This is an experience report about the performance of a workshop with nursing staff of a university hospital. Participants in the workshop were 60 nursing staff, with six workstations for the Promotion of Quality of Life at Work. Six workstations were carried out, simultaneously, with approximately 10 workers in each and an average duration of 30 minutes. The stations were conducted by nurses and physiotherapists with the support of a professor, covering themes relevant to the Health of the worker. The report aims to contribute to broadening the discussions on the subject, offering subsidies to rethink the work process and possible interventions aimed at improving working conditions, empowering workers in relation to selfcare and preventing accidents and diseases.
\end{abstract}

DESCRIPTORS: Nursing; Health of the worker; Health promotion.

\section{PROMOCIÓN DE LA CUALIDAD DE VIDA EN EL TRABAJO: RELATO DE EXPERIENCIA DE UN WORKSHOP CON TRABAJADORES DE ENFERMERÍA}

RESUMEN: El objetivo del estudio fue describir la experiencia de la realización de un workshop para la Promoción de la Cualidad de Vida en el Trabajo con trabajadores de enfermería. Es un relato de experiencia acerca de la realización de un workshop con trabajadores de enfermería de un hospital universitario. Participaron del workshop 60 trabajadores de enfermería en seis estaciones de trabajo destinadas a la Promoción de la Cualidad de Vida en el Trabajo. Fueron realizadas seis estaciones de trabajo, de modo simultáneo, con aproximadamente 10 trabajadores en cada una y duración media de 30 minutos. Las estaciones fueron conducidas por enfermeros y fisioterapeutas con el apoyo de docente y temáticas relevantes para la Salud del trabajador. El relato pretende contribuir para ampliación de las discusiones acerca del tema, ofrecendo subsidios para repensar el proceso de trabajo y las posibles intervenciones a fin de mejorar las condiciones de trabajo, empoderamiento de los trabajadores acerca del autocuidado y prevención de accidentes y enfermedades.

DESCRIPTORES: Enfermería; Salud del trabajador; Promoción de la salud. 


\section{INTRODUÇÃO}

A problemática da saúde do trabalhador tem sido foco de estudos nacionais e internacionais, evidenciando a necessidade de intervenções ${ }^{(1-4)}$. $\mathrm{Na}$ Enfermagem, autoras descrevem a exposição dos trabalhadores de enfermagem às cargas biológicas, químicas, físicas, mecânicas, fisiológicas e psíquicas no trabalho, fundamentadas no referencial da determinação social, gerando processos de adaptação que se traduzem em desgaste $^{(2,5)}$. Os processos de desgaste podem ser definidos como a perda da capacidade potencial e/ou efetiva do corpo e da mente do trabalhador, ou o próprio adoecimento, com consequente presenteísmo, absenteísmo e perda da produtividade ${ }^{(6)}$. Nesse aspecto, as doenças do sistema osteomuscular e os transtornos mentais e comportamentais, representam os grupos de doenças que mais geram dias de afastamento e incapacidade temporária ou permanente nos trabalhadores de enfermagem.

Apesar da magnitude desta problemática, a subnotificação de acidentes do trabalho situase em torno de $75 \%$ a $85 \%$ dos casos, e tanto os problemas musculoesqueléticos e quanto os de natureza psíquica nos trabalhadores, têm grande impacto na qualidade da assistência de enfermagem $^{(7)}$. Nas instituições de saúde, as incapacidades para o trabalho vêm ocasionando um ciclo grave de adoecimento nos trabalhadores, considerando a penosidade, as cargas psíquicas e a dificuldade de reposição da força de trabalho em termos quantitativos e qualitativos ${ }^{(7)}$.

Diante da problemática em questão, faz-se necessária a adoção de medidas de intervenção com vistas à promoção da saúde e da qualidade de vida do trabalhador e melhoria das condições de trabalho. A promoção da saúde propõe a articulação de saberes técnicos e populares bem como a mobilização de recursos institucionais e comunitários públicos e privados para a sua resolução. Este conceito está relacionado a diversos outros como a qualidade de vida, saúde, equidade, democracia, cidadania, entre outros. Além disso, salienta-se que são necessárias várias estratégias e articulações, sejam individuais, comunitárias, realizadas pelo Estado e pelo sistema de saúde para que ocorram ações de promoção à saúde ${ }^{(8)}$. Nesse contexto, entre as estratégias priorizadas pela Promoção à Saúde, merecem destaque a constituição de políticas públicas, a criação de ambientes sustentáveis, a reorientação dos serviços de saúde, o desenvolvimento da capacidade dos sujeitos individuais por meio do empoderamento e o fortalecimento de ações comunitárias.

Como alicerce destas estratégias, encontram-se princípios que atuam nos determinantes e causas da saúde, da participação social e da necessidade de elaboração de alternativas às práticas educativas que se restringem à intervenção sobre os hábitos e estilos de vida individuais ${ }^{(9)}$. A partir da perspectiva crítico-social, preconiza-se a utilização da noção de empoderamento social/ comunitário por considerá-la capaz de contribuir para o avanço de políticas públicas intersetoriais e saudáveis com potencial político para integrar uma agenda pública em direção à cidadania, da produção de sujeitos reflexivos, autônomos e socialmente solidários.

O empoderamento pode ser definido como um processo dinâmico que envolve aspectos cognitivos, afetivos e de comportamento. Portanto, este não é realizado para as pessoas, mas sim por elas mesmas a partir do momento em que percebem uma situação de vulnerabilidade onde se apoderam de si mesmas ${ }^{(10,11)}$. Sob essa ótica, sustenta-se que os profissionais são responsáveis por criar ações ou auxiliar na criação de espaços que favoreçam os processos de empoderamento, os quais irão refletir em situações de mudança do curso de vida. Entende-se assim, que a melhoria da qualidade de vida dos trabalhadores pode ocorrer por meio das reflexões individuais provenientes de encontros coletivos que suscitem nos trabalhadores a sua conscientização acerca de suas condições de trabalho, possibilitando a reflexão e a busca de alternativas para promoção da saúde ${ }^{(11)}$.

Considerando os aspectos descritos, a proposta do presente estudo é apresentar um relato de experiência proveniente de um workshop realizado com o intuito de discutir a qualidade de vida no trabalho em hospitais universitários e sensibilizar para as questões relativas ao processo de adoecer com vistas à melhoria das condições laborais.

O objetivo do estudo foi descrever a experiência da realização de um workshop para a promoção da qualidade de vida em trabalhadores de enfermagem de um hospital universitário de São Paulo.

\section{MÉTODO}

Trata-se do relato de experiência de um workshop realizado em um hospital universitário com vistas à promoção da qualidade de vida nos 
trabalhadores de enfermagem. A iniciativa para a realização do workshop nasceu da criação de um Grupo de Estudos sobre a Saúde dos Trabalhadores de Enfermagem no Hospital Universitário (GESTE/HU), com aprovação no Conselho Técnico para a discussão dos problemas de saúde dos trabalhadores. O workshop contou com a realização de seis estações de trabalho, a saber: "Cuidando dos meus músculos", com esclarecimentos sobre a realização adequada de movimentos no trabalho, aplicação de técnicas para relaxamento dos músculos e tendões; "Produtos químicos: que cuidados devo ter", direcionada à orientação quanto à importância do uso de Equipamentos de Proteção Individual (EPIs) na manipulação de substancias químicas e orientação sobre o uso e descarte adequado dos resíduos químicos; "Aprendendo a relaxar e reduzir o estresse", com a aplicação da técnica de relaxamento para redução da fadiga e estresse; "Distúrbios osteomusculares relacionados ao trabalho: o que eu preciso saber", que discutiu a doença e os sintomas visando à detecção precoce e tratamento adequado; "Compreendendo o desgaste psíquico no trabalhador de enfermagem", com apresentação dos conceitos do estresse, burnout e medidas que podem reduzir o desgaste psíquico no trabalho; e por fim, "Estratégias para transformar o cotidiano de trabalho", uma estação que abordou a importância da construção coletiva de uma ambiente laboral mais adequado. Participaram do workshop 60 trabalhadores de enfermagem dos turnos diurno e noturno.

\section{RESULTADOS}

O planejamento do workshop foi subdividido pelo grupo em comissões com o objetivo de divisão de tarefas para melhor organização das atividades. São elas: comissão de divulgação através de distribuição em locais estratégicos de banner e panfletos chamando a atenção para o evento, comissão responsável pela inscrição e recepção dos participantes do evento e comissão da organização do café. Além disso, a coordenadora do grupo ficou responsável pela elaboração dos certificados dos participantes e, cada componente do grupo foi selecionado em acompanhar os participantes nas estações propostas.

O workshop foi realizado no período da manhã, com a participação dos trabalhadores de enfermagem do turno da manhã, tarde e noite, totalizando 60 trabalhadores. Dentre estes estavam presentes auxiliares de enfermagem, técnicos de enfermagem, enfermeiros assistenciais e enfermeiros com cargos de chefia e diretoria. Inicialmente foi realizada uma recepção com a apresentação formal do grupo GESTE e sua finalidade na instituição e, a seguir, foram realizadas as orientações específicas sobre a dinâmica do workshop, a fim de que todos pudessem participar de todas as estações propostas. No Quadro 1 pode-se observar a descrição de cada estação.

Foram realizadas seis estações de trabalho, simultaneamente, com aproximadamente 10 trabalhadores e duração de 30 minutos. Após o término de cada estação, o grupo de 10 trabalhadores era conduzido à próxima estação por monitores, nomeados pelo GESTE-HU. As estações foram conduzidas por enfermeiros e fisioterapeutas com o apoio de um docente e a organização da infraestrutura necessária para sua execução contou com a presença dos trabalhadores de enfermagem que compõe o grupo GESTE-HU. As estações versaram sobre as temáticas de maior relevância na Saúde do trabalhador de enfermagem, viabilizando não apenas orientações e esclarecimento de dúvidas mas, a aplicação de técnicas de relaxamento para redução de fadiga e estresse. O workshop foi encerrado com uma dinâmica que envolveu todos os participantes, a fim de resgatar os conceitos de trabalho em equipe e compromisso com a melhoria das próprias condições de trabalho.

\section{DISCUSSÃO}

Com os resultados apresentados evidenciouse que a utilização da estratégia de workshop para a realização de intervenções que promovam a saúde dos trabalhadores de enfermagem é uma experiência desafiadora, e capaz de envolver diferentes níveis hierárquicos, possibilitando um espaço de aprendizado e troca de conhecimento. Estudos evidenciam a importância da construção de intervenções para a promoção da saúde do trabalhador para a redução dos agravos e para a manutenção da capacidade para o trabalho ${ }^{(1,12)}$. Nesse sentido, as estações possibilitaram a discussão acerca dos LER/DORT, que representam um dos principais problemas de saúde nos últimos tempos e são considerados como um dos agravos mais frequentes nos trabalhadores de enfermagem, uma vez que uma série de fatores inerentes à atividade assistencial contribui para o seu surgimento e alta prevalência ${ }^{(13,14,15)}$.

Esse contexto de adoecimento por problemas osteomusculares tem íntima relação com a 


\begin{tabular}{|c|c|c|c|}
\hline Estações & Palestrante & Título da Estação & Objetivos da estação \\
\hline 1 & $\begin{array}{l}\text { Terapeuta } \\
\text { integrante do GESTE/HU e } \\
\text { Mestre do Programa de Pós- } \\
\text { graduação em Gerenciamento } \\
\text { em Enfermagem/EEUSP }\end{array}$ & $\begin{array}{l}\text { Cuidando do } \\
\text { meu corpo no } \\
\text { trabalho }\end{array}$ & $\begin{array}{l}\text { Esclarecimentos e orientações sobre a } \\
\text { realização adequada de movimentos } \\
\text { no trabalho e aplicação de técnicas para } \\
\text { relaxamento dos músculos e tendões }\end{array}$ \\
\hline 2 & $\begin{array}{l}\text { ConvidadadaEEUSP, Enfermeira, } \\
\text { Doutora do Programa de Pós- } \\
\text { graduação em Gerenciamento } \\
\text { em Enfermagem/EEUSP }\end{array}$ & $\begin{array}{l}\text { P r o d u t o s } \\
\text { químicos: que } \\
\text { cuidados devo } \\
\text { ter? }\end{array}$ & $\begin{array}{l}\text { Direcionada à orientação quanto } \\
\text { à importância do uso de EPIs na } \\
\text { manipulação de substâncias químicas } \\
\text { e orientação sobre o uso e descarte } \\
\text { adequado dos resíduos químicos }\end{array}$ \\
\hline 3 & $\begin{array}{l}\text { Fisioterapeuta, integrante } \\
\text { GESTE/HU e } \\
\text { EEUSP }\end{array}$ & $\begin{array}{l}\text { Aprendendo a } \\
\text { relaxar e reduzir } \\
\text { o estresse }\end{array}$ & $\begin{array}{l}\text { Aplicação da técnica de relaxamento } \\
\text { muscular progressivo de Jacobson para } \\
\text { redução da fadiga e estresse }\end{array}$ \\
\hline 4 & $\begin{array}{l}\text { ConvidadadaEEUSP, Enfermeira, } \\
\text { Mestre do Programa de Pós- } \\
\text { graduação em Gerenciamento } \\
\text { em Enfermagem/EEUSP }\end{array}$ & $\begin{array}{l}\text { DORT: o que eu } \\
\text { preciso saber? }\end{array}$ & $\begin{array}{l}\text { Discutiu a doença, os sintomas visando } \\
\text { à detecção precoce e tratamento } \\
\text { adequado. }\end{array}$ \\
\hline 5 & $\begin{array}{l}\text { Convidada daEEUSP, Enfermeira, } \\
\text { Mestranda do Programa de Pós- } \\
\text { graduação em Gerenciamento } \\
\text { em Enfermagem/EEUSP }\end{array}$ & $\begin{array}{l}\text { O desgaste } \\
\text { psíquico no } \\
\text { trabalhador de } \\
\text { enfermagem }\end{array}$ & $\begin{array}{l}\text { Compreender e apresentação dos } \\
\text { conceitos do estresse, burnout e } \\
\text { medidas que podem reduzir o desgaste } \\
\text { psíquico no trabalho. }\end{array}$ \\
\hline 6 & $\begin{array}{l}\text { Enfermeira, integrante do } \\
\text { GESTE/HU, Docente Associada } \\
\text { do Departamento de Orientação } \\
\text { Profissional da EEUSP }\end{array}$ & $\begin{array}{l}\text { Estratégias para } \\
\text { transformar } \mathrm{O} \\
\text { cotidiano de } \\
\text { trabalho }\end{array}$ & $\begin{array}{l}\text { Abordou a importância da construção } \\
\text { coletiva de um ambiente laboral mais } \\
\text { adequado. }\end{array}$ \\
\hline
\end{tabular}

rotina de trabalho, caracterizada pelo ritmo intenso, manuseio de equipamentos, materiais obsoletos e danificados, entre outros ${ }^{(6,16,17)}$. Também foi possível discutir a dificuldade entre os trabalhadores para encontrar tratamentos efetivos que minimizem os sintomas, os quais podem influenciar na incapacidade, impactando na realização das atividades diárias e de trabalho, e que, se não tratados adequadamente, resultam em redução da qualidade de vida e de saúde, gerando custos com a saúde ${ }^{(18,19)}$.

A estação de trabalho que abordou o desgaste psíquico buscou resgatar as queixas mais relatadas por estes trabalhadores que têm relação com a pressão e controle, supervisão estrita, muito presentes ainda no processo de trabalho de enfermagem, ocasionando problemas como os transtornos mentais e comportamentais, além de sintomas como enxaqueca, distúrbios gástricos e insônia ${ }^{(20)}$. Portanto, concretizouse como um espaço reflexivo e de diálogo, onde os trabalhadores puderam conhecer os conceitos, discutir sobre os sintomas e perceber a necessidade de atentarem para as demandas psíquicas no processo de trabalho. Esta estação também trouxe à tona sentimentos variados de conquistas e frustrações, em decorrência do cotidiano vivencial dos trabalhadores, favorecendo a troca de experiências acerca do sofrimento psíquico. Alguns estudos apontam que há uma relação entre o sofrimento psíquico dos trabalhadores de enfermagem e o processo de trabalho em âmbito hospitalar. E, que há a necessidade de traçar intervenções para a identificação e tratamento. Essas estratégias podem ser a nível organizacional, aplicadas ao ambiente de trabalho; estratégias individuais focadas nas respostas pessoais, ao tentar regular as emoções frente a uma situação estressante; estratégias combinadas centradas na interação do contexto ocupacional com o indivíduo. As ações preventivas serão viáveis quando o sofrimento psíquico não for responsabilidade única e um estigma voltado para o profissional-usuário e, sim um problema da relação indivíduo-processo de trabalho-organização ${ }^{(21,22)}$. Considerando que os trabalhadores de enfermagem frequentemente referem dor, incapacidade, além de sintomas 
de ansiedade e estresse, ações para evitar sua progressão são de grande importância, pois interferem na qualidade de vida dos profissionais. Nesse aspecto, as práticas de relaxamento foram conduzidas de modo a sensibilizar e instrumentalizar o trabalhador para seu autocuidado. Uma das realizações no evento em uma das estações foi aplicar e ensinar técnicas de relaxamento dos músculos e tendões e, segundo um estudo que avaliou a capacidade funcional do trabalhador revelou que atividade física auxilia na melhora da capacidade funcional ${ }^{(23)}$. Outro estudo da Finlândia corrobora com essa intervenção mostrando que um dos pontos positivos que influenciam na capacidade do trabalho, na habilidade e competência do trabalhador é a atividade física, tendo uma repercussão na qualidade de $v i_{a}{ }^{(24)}$. Pesquisas reafirmam a necessidade de desenvolver estratégias de cuidado com os trabalhadores de enfermagem. As realizações de técnicas de relaxamento apresentam resultados significativos na redução da dor musculoesquelética e do estresse, durante e após a jornada de trabalho, não devendo ser adotadas como os únicos métodos preventivos e de promoção da saúde no trabalho( ${ }^{(25,26)}$.

Outro fator discutido na literatura é o envelhecimento do trabalhador associado a demandas do trabalho. Vários estudos corroboram com essa associação descrevendo que as instituições não estão preparadas para conviver com o envelhecimento dos profissionais, pois, além dos profissionais de enfermagem que trabalham em hospitais ficarem expostos constantemente a situações de estresse físico e emocional, eles estão envelhecendo ocasionando também uma aposentadoria precoce ${ }^{(27,28,29)}$.

Uma das estações abordou a importância do uso de equipamentos de proteção na manipulação de substâncias químicas e orientação sobre o uso e descarte correto dos resíduos químicos, possibilitando maior conhecimento aos trabalhadores e sensibilização acerca da problemática. Pesquisas evidenciam a exposição à carga química entre os trabalhadores de enfermagem, com inúmeros agravos decorrentes dessa exposição, resgatando a necessidade de um plano de intervenções com o objetivo de prevenir e promover a saúde dos trabalhadores de enfermagem nessa questão ${ }^{(2)}$.

O encerramento do workshop possibilitou a discussão de temas como autonomia, trabalho em equipe e a corresponsabilidade de todos, por meio de uma dinâmica envolvendo todos os trabalhadores de enfermagem. Essas discussões proporcionam um espaço para a reflexão sobre a autonomia, responsabilidade e trabalho em equipe, possibilitando aos trabalhadores o repensar da prática laboral e a qualidade de vida, por meio do empoderamento e a criação de processosreflexivos, tornando-sesujeitospróprios da mudança. Nesse aspecto, a problemática de saúde do trabalhador de enfermagem tornase um desafio para as instituições de saúde e o gerenciamento de recursos humanos em enfermagem. No contexto atual, a incorporação de estratégias de intervenção nos ambientes de trabalho deve fazer parte da missão institucional, tendo como prioridade a gestão de recursos humanos, considerando o impacto na qualidade dos processos de trabalho quando o trabalhador está saudável e realizado com a sua atividade profissional.

\section{CONCLUSÃO}

A realização do workshop evidenciou as lacunas do conhecimento sobre o adoecer no trabalho, e possibilitou a sensibilização dos trabalhadores para a construção coletiva de um ambiente laboral mais salutar. As estações versaram sobre os temas de maior relevância, considerando os agravos presentes nos trabalhadores de enfermagem e possibilitaram além do espaço para debate e orientação, a troca de conhecimento e de experiências sobre o processo de trabalho. Salienta-se, com esta experiência, a responsabilidade de cada grupo na identificação e na construção de estratégias criativas que possam envolver os trabalhadores, minimizando os acidentes e as doenças relacionadas ao trabalho, oferecendo subsídios para novas pesquisas, sobretudo de caráter interventivo no campo da saúde do trabalhador.

\section{REFERÊNCIAS}

1. Silva SM, Baptista PCP, Felli VEA, Caldas AM, Sarquis LMM, Mininel VA. Intervention strategies for the health of university hospital nursing staff in Brazil. Rev. Latino-Am. Enfermagem. 2013; 21(1): 300-308.

2. Costa TF, Felli VEA,Baptista PCP.A percepção dos trabalhadores de enfermagem sobre o manejo dos resíduos químicos perigosos. Rev.esc.enferm. USP vol 46no.6 São Paulo Dec.2012.

3. Consonni D, Marinaccio A, Bertazzi PA, Iavicoli S. Occupational health. Work related injuries decreasing, occupational diseases increasing. Epidemiol Prev; 2011; 35(5-6 Suppl 2): 106-7.

4. Loisel P, Lemaire J, Poitras S, Durand MJ, Champagne 
F, Stock S et al. Cost-benefit and cost-effectiveness analysis of a disability prevention model for pain management: a six year follow up study. Occup Environ Med. 2002; 59:807-15.

5. Costa TF, Felli VEA. Gerenciamento de resíduos químicos perigosos manuseados pela enfermagem de um hospital universitário. [tese]. São Paulo. Escola de Enfermagem da Universidade de São Paulo; 2009.

6. Silva, FJ. A capacidade para o trabalho e a fadiga entre trabalhadores de enfermagem em unidades de internação médica e cirúrgica [dissertação]. São Paulo. Escola de Enfermagem da Universidade de São Paulo; 2011.

7. Baptista PCP. Pesquisa-ação como estratégia de promoção à saúde dos trabalhadores de enfermagem em cenário nacional. Relatório Final de Pesquisa, 2014.

8. Buss, PM. Promoção da saúde e qualidade de vida. Ciência \& Saúde Coletiva. 2000; 5(1):163-177.

9. Carvalho SR, Gastaldo D. Promoção à saúde e empoderamento: uma reflexão a partir das perspectivas crítico-social pós-estruturalista. Ciência \& Saúde Coletiva.2008;13(2):2029-2040.

10. Kleba ME, Wendausen A. Empoderamento: processo de fortalecimento dos sujeitos nos espaços de participação social e democratização política. Saúde Soc. 2009; 18(4):733-743.

11. Carvalho RS, Os múltiplos sentidos da categoria "empowerment" no projeto de Promoção à Saúde. Cad. Saúde Pública. 2004; 20(4):1088-1095.

12. Duran ECM; Cocco MIM. Capacidade para o trabalho entre trabalhadores de enfermagem do pronto-socorro de um hospital universitário. Revista Latino Americana de Enfermagem.2004 janeirofevereiro:12(1):43-9.

13. Salerno VL, Silvestre MP, Sabino MO. Interfaces LER/Saúde Mental: a experiência de um Centro de Referência em Saúde do trabalhador do Estado de São Paulo. Rev. Bras. Saude Ocup. 2011;36(123):128-38.

14. Magnano TSBS, Lisboa MTL, Griep RH. Trabalho da enfermagem e distúrbio musculoesquelético: revisão das pesquisas sobre o tema. Escola Anna Nery Revista de Enfermagem. 2008; 12(3):560-65.

15. Alexandre NMC. Ergonomia e as atividades ocupacionais da equipe de enfermagem. Rev. Esc. Enferm. USP. 1998; 32(1):84-90.

16. Cavalcanti FMS et al. IN: Souza DO, Silva SEV. Enfermagem em diversas áreas de atuação. Maceió: Edufal, 2012.

17. Ferrari AL. Adaptação transcultural do questionário "cultural study of musculo-skeletal and other symptoms and associated disability" CUPID Questionnaire. São Paulo. 2009.

18. Cunha AC et al. Effect of global posture reeducation and of static stretching com pain, range of motion, and quality of life in women with chronic nedk pain: a randomized clinical Trial. Clinics, São Paulo. 2008;63:763-770.

19. Kessler RC, Ustun TB. The world mental health (WMH) survey initiative version of the world health organization (WHO) composite international diagnostic interview (CIDI). Internet. Journal of Methods in Psychiatric Research, Chichester. 2004;13:93-121.

20. Vasconcelos LHS. Avaliação da eficácia da técnica de relaxamento muscular progressivo de Jacobson na redução de fadiga em uma trabalhadora de enfermagem. [dissertação]. São Paulo. Escola de Enfermagem da Universidade de São Paulo; 2014.

21. Tito RS. Burnout e Transtornos Mentais comum nos trabalhadores que assistem crianças com cardiopatias grave.Dissertação.São Paulo: Escola de Enfermagem da Universidade de São Paulo. São Paulo; 2013.

22. Moreno NF,Gil GP,Haddad MCL, Vannuchi MTO. Estratégias e intervenções no enfrentamento da Síndrome de Burnout. Rev.enf.UERJ, Rio de Janeiro, 2011 jan/mar; 19(1): 140-5.

23. Raffone AM, HenningtonEA.Avaliação dacapacidade funcional dos trabalhadores de enfermagem. Rev. Saúde Pública. 2005; 39(4):669-76.

24. Tuomi K, Huuhtanen P, Nykyri E, Ilmarinem J.Promotion of work ability, the quality of work and retirement. -Occup.Med.2001; 51: 318-324.

25. Ferracini GN, Valente FM. Presença de sintomas musculoesqueléticos e efeitos da ginástica laboral em funcionários do setor administrativo de um hospital público. Rev. Dor. 2010;11(3).

26. Sancinetti, TR, Gaidzinski RR, Felli VEA, Fugulin FMT, Baptista PCP, Ciampone MHT, Kurcgant P, Silva FJ. Absenteísmo: doença na equipe de enfermagem: relação com a taxa de ocupação. Rev. Esc. Enferm. USP [online]. 2009;43(2):1277-1283.

27. Alves F, Fleury MT. Envelhecimento da força de trabalho no Brasil. Parceria com a EAESP-FGV.Apoio Fiesp, Sesi e Amcham. Março 2013.

28. Sampaio RF, Augusto VG. Envelhecimento e trabalho: um desafio para a agenda de reabilitação. Rev.bras.fisioter.2012;16(2).

29. França LHFP. O envelhecimento populacional e seu reflexo nas organizações: A importância da Educação ao longo da vida. Téc.Senac:a R.Educ.Prof.2011;37(2). 